PROCEEDINGS OF THE

AMERICAN MATHEMATICAL SOCIETY

Volume 137, Number 9, September 2009, Pages 3099-3103

S 0002-9939(09)09940-7

Article electronically published on April 30, 2009

\title{
ESTIMATING THE TRACE-FREE RICCI TENSOR IN RICCI FLOW
}

\author{
DAN KNOPF
}

(Communicated by Matthew J. Gursky)

\begin{abstract}
An important and natural question in the analysis of Ricci flow behavior in all dimensions $n \geq 4$ is this: What are the weakest conditions that guarantee that a solution remains smooth? In other words, what are the weakest conditions that provide control of the norm of the full Riemann curvature tensor? In this short paper, we show that the trace-free Ricci tensor is controlled in a precise fashion by the other components of the irreducible decomposition of the curvature tensor, for all compact solutions in all dimensions $n \geq 3$, without any hypotheses on the initial data.
\end{abstract}

\section{INTRODUCTION}

It is common and indeed expected that the solutions of Ricci flow will encounter finite-time singularities. For example, a solution of Ricci flow on a compact manifold whose scalar curvature is bounded from below by $r>0$ at $t=0$ must become singular at or before the formal vanishing time $T_{\text {form }}=\frac{n}{2 r}$. Consequently, the following is a natural and important question:

Question 1.1. What is the simplest quantity that, when controlled, guarantees that a solution of Ricci flow will remain smooth up to time $T$ ?

A solution $\left(\mathcal{M}^{n}, \omega(\cdot)\right)$ of Kähler-Ricci flow remains smooth until the volume of an analytic subvariety goes to zero. Indeed, work of Gang Tian and Zhou Zhang 11 proves that a smooth solution exists as long as the cohomology class $[\omega]$ remains Kähler, while results of Jean-Pierre Demailly and Mihai Paun 4 show that this can happen only if there exists an analytic subvariety whose volume is forced to vanish.

For general (i.e., non-Kähler) solutions of Ricci flow, the picture is less clear. Standard short-time existence results imply that a solution $\left(\mathcal{M}^{n}, g(\cdot)\right)$ of Ricci flow on a compact manifold becomes singular at $T<\infty$ if and only if

$$
\lim _{t \nearrow T}\left(\max _{x \in M^{n}}|\operatorname{Rm}(x, t)|\right)=\infty .
$$

Thus the question posed above can be recast more precisely as follows:

Question 1.2. What are the weakest conditions that provide control of the norm of the full Riemann curvature tensor on a solution of Ricci flow?

Received by the editors July 14, 2008.

2000 Mathematics Subject Classification. Primary 53C44, 58J35.

The author acknowledges NSF support in the form of grants DMS-0545984 and DMS-0505920.

(C)2009 by the author 
In any dimension, it is true that a finite-time singularity occurs on a compact manifold if and only if

$$
\limsup _{t \nearrow T}\left(\max _{x \in M^{n}}|\operatorname{Rc}(x, t)|\right)=\infty .
$$

Nataša Šešum has given a direct proof $[9$. The result also follows from independent results of Miles Simon [10] by a short argument, which we reproduce here for the convenience of the reader. Assume that $g(t)$ is smooth for $t \in[0, T)$, where $T<\infty$. If limsup $\operatorname{su}_{t>T}\left(\max _{x \in \mathcal{M}^{n}}|\operatorname{Rc}(x, t)|\right)<\infty$, then [5, Lemma 14.2] guarantees existence of a complete $C^{0}$ limit metric $g(T)$. One may then apply [10, Theorem 1.1], choosing a background metric $\bar{g}:=g(T-\delta)$ such that $(1-\varepsilon) \bar{g} \leq g \leq(1+\varepsilon) \bar{g}$, where $\varepsilon=\varepsilon(n)$ and $\delta=\delta(\varepsilon)$. Let $\bar{K}=\max _{x \in \mathcal{M}^{n}}|\operatorname{Rm}(\bar{g})|_{\bar{g}}$. Simon's theorem implies that there exists $\eta=\eta(n, \bar{K})$ such that for any $\theta \in[0, \delta]$, a solution $\hat{g}(s)$ of harmonic-mapcoupled Ricci flow exists for $0 \leq s<\eta$ and satisfies $\hat{g}(0)=g(T-\theta)$; moreover, $\hat{g}(s)$ is smooth for $0<s<\eta$. Because harmonic-map-coupled Ricci flow is equivalent to Ricci flow modulo diffeomorphisms, the result follows by taking $\theta=\eta / 2$.

In dimension three, an eminently satisfactory answer to Question 1.2 is given by the important pinching theorem obtained independently by Thomas Ivey [7] and Richard Hamilton [6]. Their estimate implies in particular that the scalar curvature dominates the full curvature tensor of any Ricci flow solution on a compact 3-manifold with normalized initial data. (As is well known, the Hamilton-Ivey pinching estimate implies the much stronger result that any rescaled limit of a finite time singularity in dimension three must have nonnegative sectional curvature.)

Xiuxiong Chen has conjectured that an appropriate bound on scalar curvature alone might be sufficient to rule out singularity formation in all dimensions. Partial progress toward this conjecture was made recently by Bing Wang 12. He proves that if the Ricci tensor is uniformly bounded from below on $[0, T)$ and if an integral bound

$$
\int_{0}^{T} \int_{\mathcal{M}^{n}}|R|^{\alpha} d \mu d t<\infty
$$

holds for some $\alpha \geq(n+2) / 2$, then no singularity occurs at time $T<\infty$. This is optimal in the sense that the example of a shrinking round sphere suggests that no smaller value of $\alpha$ can suffice. However, Wang's result requires the restrictive hypothesis that $\inf _{\mathcal{M}^{n} \times[0, T)} \mathrm{Rc} \geq-A>-\infty$. Ricci flow does not in general preserve nonnegative Ricci curvature in dimensions $n \geq 4$. See [8] for examples starting in dimension $n=4$ and work of Christoph Böhm and Burkhard Wilking 2 for compact examples starting in dimension $n=12$. Moreover, examples of local singularity formation [1] show that one cannot expect a bound like Rc $\geq-A$ to be preserved in general. For these reasons, one is strongly motivated to study the extent to which the scalar curvature may control the trace-free Ricci tensor in general.

Recall that in any dimension $n \geq 3$, the Riemann curvature tensor admits an orthogonal decomposition

$$
\mathrm{Rm}=U+V+W
$$

into irreducible components

$$
U=\frac{1}{2 n(n-1)} R(g \otimes g), \quad V=\frac{1}{n-2}(F \otimes g), \quad W=\text { Weyl tensor },
$$


where $\otimes$ denotes the Kulkarni-Nomizu product of symmetric tensors and $F$ denotes the trace-free Ricci tensor. The purpose of this short paper is to observe that $V$ is always dominated by the other components in the following sense:

Main Theorem. If $\left(\mathcal{M}^{n}, g(\cdot)\right)$ is a solution of Ricci flow on a compact manifold of dimension $n \geq 3$, then there exist constants $c\left(g_{0}\right) \geq 0, C_{1}\left(n, g_{0}\right)>0$, and $C_{2}(n)>0$ such that for all $t \geq 0$ such that a solution exists, one has $R+c>0$ and

$$
\frac{|V|}{R+c} \leq C_{1}+C_{2} \max _{s \in[0, t]} \sqrt{\frac{|W|_{\max }(s)}{R_{\min }(s)+c}} .
$$

This result educes further questions about the relationship (if any) between $R$ and $|W|$ along solutions of Ricci flow. In this direction, Böhm and Wilking have recently announced [3] that the condition $R \geq c_{n}|W|$ defines an invariant curvature cone for Ricci flow in all sufficiently high dimensions, where $c_{n}=\sqrt{2(n-1)(n-2)}$ if $n$ is even and $c_{n}=\sqrt{2(n-1)(n-2)}+o(1)$ as $n \rightarrow \infty$ if $n$ is odd. This is a strong hypothesis: for example, if $\left(\mathcal{M}^{n}, g\right)$ is an Einstein manifold of positive scalar curvature with $R \geq c_{n}|W|$, the Böhm-Wilking results imply that its universal cover is isometric to one of $\mathcal{S}^{n}, \mathcal{S}^{\frac{n}{2}} \times \mathcal{S}^{\frac{n}{2}}(n$ even $)$, or $\mathcal{S}^{\frac{n-1}{2}} \times \mathcal{S}^{\frac{n+1}{2}}$ ( $n$ odd), with their canonical metrics.

Whether the scalar curvature controls the norm of the Weyl tensor in some general sense under Ricci flow (i.e. for solutions whose initial data lie outside the $R \geq c_{n}|W|$ cone) remains a highly interesting open question.

\section{Proof of the Main theorem}

Define

$$
a=|F|=\frac{\sqrt{n-2}}{2}|V|,
$$

noting that $a$ is smooth wherever it is strictly positive. Choose $c \geq 0$ large enough so that $R_{\min }(0)+c>0$ and define

$$
b=R+c,
$$

noting that $b>0$ for as long as a solution exists.

In any dimension $n \geq 3$, one has

$$
\frac{\partial}{\partial t}|F|^{2}=\Delta|F|^{2}-2|\nabla F|^{2}+\frac{4(n-2)}{n(n-1)} R|F|^{2}-\frac{8}{n-2} \operatorname{tr} F^{3}+4 W(F, F),
$$

where $\operatorname{tr} F^{3}=F_{i}^{j} F_{j}^{k} F_{k}^{i}$ and $W(F, F)=W_{i j k \ell} F^{i \ell} F^{j k}$. It follows from CauchySchwarz that $a$ obeys the differential inequality

$$
a_{t} \leq \Delta a+\frac{2(n-2)}{n(n-1)} a(b-c)-\frac{4}{n-2} a^{-1} \operatorname{tr} F^{3}+2 a^{-1} W(F, F) .
$$

The positive quantity $b$ evolves by

$$
b_{t}=\Delta b+2 a^{2}+\frac{2}{n}(b-c)^{2} .
$$

To prove the theorem, it will suffice to bound the scale-invariant nonnegative quantity

$$
\varphi=\frac{a}{b}
$$


Because $\Delta \varphi=b^{-1}(\Delta a-\varphi \Delta b)-2\langle\nabla \varphi, \nabla \log b\rangle$, one has

$$
\varphi_{t} \leq \Delta \varphi+2\langle\nabla \varphi, \nabla \log b\rangle+2 \rho \varphi
$$

where the reaction term is

$$
\rho=\frac{n-2}{n(n-1)}(b-c)-\frac{2}{n-2} \frac{\operatorname{tr} F^{3}}{a^{2}}+\frac{W(F, F)}{a^{2}}-\frac{(b-c)^{2}}{n b}-a \varphi .
$$

There exist positive constants $c_{1}, c_{2}$ depending only on $n \geq 3$ such that

$$
\left|\frac{2}{n-2} \operatorname{tr} F^{3}\right| \leq c_{1} a^{3} \quad \text { and } \quad|W(F, F)| \leq c_{2}|W| a^{2} .
$$

Hence

$$
\rho \leq \frac{n-2}{n(n-1)}(b-c)+c_{1} a+c_{2}|W|-\frac{(b-c)^{2}}{n b}-a \varphi .
$$

Define constants $\alpha, \beta, \gamma$ by $\alpha^{2}=c_{1}, \beta^{2}=\frac{n-2}{n(n-1)}$, and $\gamma^{2}=c_{2}$. Fix $\varepsilon>0$ and choose $C_{1}=\max \left\{\alpha^{2}+\beta, \varphi_{\max }(0)+\varepsilon\right\}$ and $C_{2}=\gamma$. Consider the barrier function

$$
\Phi(t)=C_{1}+C_{2} \max _{s \in[0, t]} \sqrt{\frac{|W|_{\max }(s)}{b_{\min }(s)}},
$$

noting that $\Phi$ is monotone nondecreasing. If $\varphi_{\max }(t) \geq \Phi(t)$ at some $t>0$, then at any $(x, t)$ where $\varphi$ attains its spatial maximum, one has

$$
a \geq\left(\alpha^{2}+\beta\right) b+\gamma \sqrt{b|W|},
$$

which implies that

$$
\begin{aligned}
a^{2} & \geq \alpha^{2} a b+\beta^{2} b^{2}+\gamma^{2} b|W| \\
& \geq \frac{n-2}{n(n-1)}\left(b^{2}-b c\right)+\left(c_{1} a+c_{2}|W|\right) b-\frac{1}{n}(b-c)^{2},
\end{aligned}
$$

hence that $\rho \leq 0$, hence that $\varphi_{t} \leq 0$, hence that $\frac{d^{+}}{d t} \varphi_{\max }(t) \leq 0$, understood in the usual sense as the limsup of difference quotients. It follows that $\varphi_{\max }(t) \leq \Phi(t)$ for as long as a solution exists.

\section{ACKNOWLEDGEMENT}

The author warmly thanks Matt Gursky for a number of stimulating discussions.

\section{REFERENCES}

1. Angenent, Sigurd B.; Knopf, Dan. Precise asymptotics of the Ricci flow neckpinch. Comm. Anal. Geom. 15 (2007), no. 4, 773-844. MR2395258 (2009b:53106)

2. Böhm, Christoph; Wilking, Burkhard. Nonnegatively curved manifolds with finite fundamental groups admit metrics with positive Ricci curvature. Geom. Funct. Anal. 17 (2007), no. 3, 665-681. MR2346271(2008h:53050)

3. Böhm, Christoph; Wilking, Burkhard. On Ricci flow in high dimensions. In preparation.

4. Demailly, Jean-Pierre; Paun, Mihai. Numerical characterization of the Kähler cone of a compact Kähler manifold. Ann. of Math. (2) 159 (2004), no. 3, 1247-1274. MR2113021 (2005i:32020)

5. Hamilton, Richard S. Three-manifolds with positive Ricci curvature. J. Differential Geom. 17 (1982), no. 2, 255-306. MR664497 (84a:53050)

6. Hamilton, Richard S. The formation of singularities in the Ricci flow. Surveys in differential geometry, Vol. II (Cambridge, MA, 1993), 7-136, Internat. Press, Cambridge, MA, 1995. MR1375255 (97e:53075) 
7. Ivey, Thomas. Ricci solitons on compact three-manifolds. Differential Geom. Appl. 3 (1993), no. 4, 301-307. MR1249376 (94j:53048)

8. Knopf, Dan. Positivity of Ricci curvature under the Kähler-Ricci flow. Commun. Contemp. Math. 8 (2006), no. 1, 123-133. MR2208813 (2006k:53114)

9. Šešum, Nataša. Curvature tensor under the Ricci flow. Amer. J. Math. 127 (2005), no. 6, 1315-1324. MR2183526 (2006f:53097)

10. Simon, Miles. Deformation $C^{0}$ Riemannian metrics in the direction of their Ricci curvature. Comm. Anal. Geom. 10 (2002), no. 5, 1033-1074. MR.1957662 (2003j:53107)

11. Tian, Gang; Zhang, Zhou. On the Kähler-Ricci flow on projective manifolds of general type. Chinese Ann. Math. Ser. B 27 (2006), no. 2, 179-192. MR2243679 (2007c:32029)

12. Wang, Bing. On the conditions to extend Ricci flow. Int. Math. Res. Not. (2008), Article ID rnn012, 30 pp. MR2428146

Department of Mathematics, University of Texas at Austin, Austin, Texas 78712-0257

E-mail address: danknopf@math.utexas.edu

$U R L:$ http://www.ma.utexas.edu/users/danknopf/ 